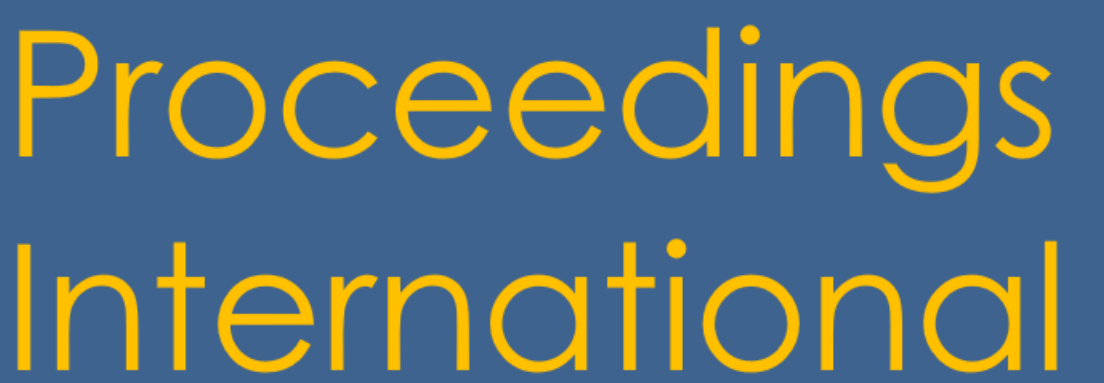

Volume 1, Issue 1, Pages 0013-0014

\title{
Microstructure investigation of r.f. current annealed FINEMET cold drawn microwires by UHR-TEM
}

\author{
G. Ababei 1,* , A. Damian ${ }^{1}$, G. Stoian ${ }^{1}$, V. Dobrea ${ }^{1}$, S. Corodeanu ${ }^{1}$, N. Lupu ${ }^{1}$, H. Chiriac ${ }^{1}$ \\ 1 National Institute of Research \& Development for Technical Physics, 47 Mangeron Boulevard, Iasi, ROMANIA \\ * Correspondence: gababei@phys-iasi.ro; Scopus ID: 56835895100
}

The microstructure of FINEMET $\left(\mathrm{Fe}_{73.5} \mathrm{Nb}_{3} \mathrm{Cu}_{1} \mathrm{Si}_{13.5} \mathrm{~B}_{9}\right.$, at \%) ferromagnetic microwires with diameters of $30 \mu \mathrm{m}$ obtained by cold drawn of $120 \mu \mathrm{m}$ thicker conventional amorphous microwires followed by radio frequency current annealing has been investigated by ultra-high resolution transmission electron (UHR-TEM) microscopy.

The cold drawn microwires (CDWs) were annealed by radio frequency current of 375 $\mathrm{mA} @ 10 \mathrm{MHz}$ and $750 \mathrm{~mA} @ 10 \mathrm{MHz}$, respectively, for 10 minutes to achieve cold drawn microwires with nano-crystalline structure.

The specimens for UHR-TEM investigation were prepared in the shape of lamella using Focus Ion Beam microscope. For each annealed CDNWs were obtained two lamella one from the edge of microwire and another one from the center of the wire. Each specimen has been examined by transmission electron microscopy (TEM) using a Libra200MC microscope operated at 200kV through the bright-field and dark-field TEM, HR-TEM, HAADF STEM and EDX method. Electron diffraction patterns are interpreted using the computer programs SingleCrystal ${ }^{\circledR}$ and $\mathrm{JEMS}^{\circledR}$. Mean grain size is measured using ImageJ software and making a geometrical correction for film thickness.

The cold drawn microwires annealed at $350 \mathrm{~mA}$ are largely amorphous, although containing occasional $\mathrm{Fe}_{3} \mathrm{Si}$ regions of short-range order as presented in Figure 1 (a).

The cold drawn microwires annealed at $750 \mathrm{~mA}$ present a nano-crystalline structures, with all nano-grains appearing to be of the bcc $\alpha-\mathrm{Fe}(\mathrm{Si})$ structure as shown in Figure 1 (b). The estimated number density of nano-grains is $4.8 \times 10^{23}$, which corresponds to an estimated phase fraction of $25 \mathrm{vol} . \%$ for a nano-grain radius of $5 \mathrm{~nm}$.

Line-scan EDX shows no particularly striking deviation from a homogenous distribution of elements for nanocrystalline sample, while a clustering behavior of elements for amorphous sample was observed.

Keywords: FINEMET, microwires, UHR-TEM, Libra200MC. 


\section{G. Ababei, A. Damian, G. Stoian, V. Dobrea, S. Corodeanu, N. Lupu, H. Chiriac}

\section{Funding}

Not applicable.

\section{Acknowledgments}

Work supported by the Romanian Ministry of Research and Innovation (MCI) under NUCLEU Program - contract no. 33N/2019, project PN 19280101 and Contract No.11PFE/16.10.2018.

\section{Conflicts of Interest}

The authors declare no conflict of interest.
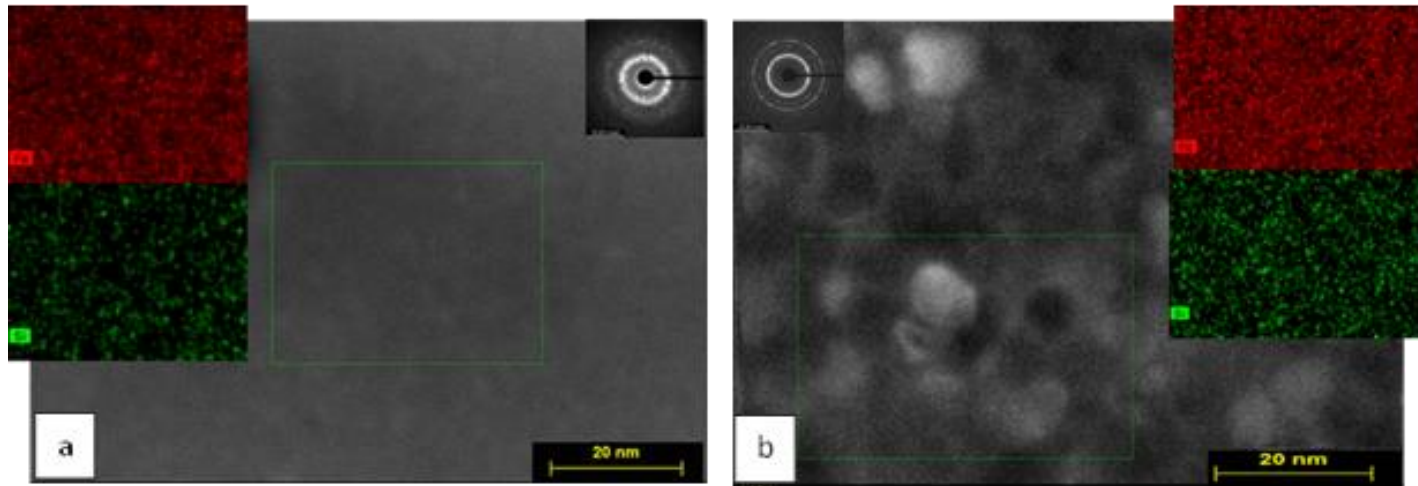

Figure 1. Microstructure of CDWs annealed at $375 \mathrm{~mA}$ (a) and $750 \mathrm{~mA}$ (b) r.f. current.

Inset shows the EDX mapping of Fe (red) and $\mathrm{Si}$ (green) atoms in the CDWs microstructure.

(C) 2019 by the authors. This article is an open access article distributed under the terms and conditions of the Creative Commons Attribution (CC BY) license (http://creativecommons.org/licenses/by/4.0/). 